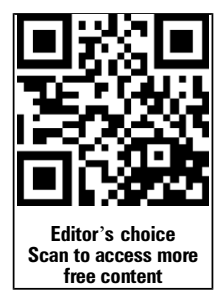

${ }^{1}$ Neuroradiology Research Laboratory, Mayo Clinic, Rochester, Minnesota, USA ${ }^{2}$ Center for Computational Fluid Dynamics, College of Sciences, George Mason University, Fairfax, Virginia, USA

\section{Correspondence to} Dr R Kadirvel, Neuroradiology Research Laboratory, Mayo Clinic, 200 First St SW, Rochester, MN 55905, USA: kadir@mayo.edu

Received 4 September 2014 Revised 25 September 2014 Accepted 29 September 2014 Published Online First 20 October 2014

\title{
Gene expression comparison of flow diversion and coiling in an experimental aneurysm model
}

\author{
Cole Puffer, ${ }^{1}$ Daying Dai, ${ }^{1}$ Yong-Hong Ding, ${ }^{1}$ Juan Cebral, ${ }^{2}$ David Kallmes, \\ Ramanathan Kadirvel ${ }^{1}$
}

\begin{abstract}
Background and purpose Mechanisms of both healing and complications, including spontaneous aneurysm rupture, remain unclear following flow diverter treatment. The aim of this study was to compare gene expression of various key molecules involved in the healing of aneurysms, between aneurysms treated with microcoils and flow diverters.
\end{abstract}

Methods Saccular aneurysms were created in rabbits. Aneurysms were treated with coils $(n=6)$ or flow diverters $(n=6)$. Aneurysms were harvested at 4 weeks following treatment and used for gene expression and zymography experiments. Genes with a fold change of 1.2 or more were considered upregulated whereas those with a fold change of 0.8 or less were considered downregulated.

Results All coil embolized aneurysms were completely occluded at follow-up. Two aneurysms were occluded and the remaining four samples were incompletely occluded in the flow diverter treated group. The following genes were expressed at lower levels in the flow diverter group compared with the coiled aneurysm group: proteinases (matrix metalloproteinases 2 and 9), cellular markers (endothelial nitric oxide synthase and smooth muscle actin), and structural proteins (collagens and fibronectin). Genes related to inflammation (tumor necrosis factor $\alpha$ and monocyte chemoattractant protein 1) were upregulated in flow diverter treated aneurysms compared with coil embolized aneurysms. Notably, the enzymatic activity of active matrix metalloproteinase 9 was high in aneurysms treated with flow diverters.

Conclusions Our findings may provide improved understanding of rupture risk and healing following aneurysm treatment and inform development of therapies aimed at lowering rupture risk and accelerating healing.

\section{INTRODUCTION}

Even after nearly two decades of clinical use, long term occlusion rates with endovascular microcoils in large and broad necked aneurysms remain poor. The introduction of flow diverters in the treatment of aneurysms has alleviated the shortcomings of microcoils and has shown a remarkable rate of complete occlusion of aneurysms, which are difficult to treat using other endovascular techniques.

Both of these approaches primarily elicit thrombus formation in the aneurysm cavity and then promote neointima formation across the neck to seal the aneurysm cavity from the circulation. ${ }^{1-4}$ Coils act within the aneurysm cavity while flow diverters primarily work at the parent artery. ${ }^{5} 6$ Studies aimed at exploring the mechanism of aneurysm healing following endovascular treatments have mostly focused at the tissue and cellular levels, and a handful of preclinical experiments studied the molecular mechanisms. ${ }^{3}{ }^{7-11}$ However, the exact biological mechanism of action of these endovascular techniques remains poorly understood. Furthermore, rare but devastating reports of spontaneous aneurysm rupture following aneurysm therapy have diminished enthusiasm for flow diversion devices. ${ }^{12}{ }^{13}$ The mechanisms underlying the propensity for aneurysm rupture following flow diversion treatment remain enigmatic.

The aim of the current study was to directly compare gene expression of key molecules potentially involved in the healing of aneurysms as well as the activity of proteolytic enzymes, between aneurysms treated with microcoils and flow diverters.

\section{MATERIALS AND METHODS}

Aneurysm creation, treatment, and follow-up

The Institutional Animal Care and Use Committee approved all procedures before initiation of the study. Elastase induced saccular aneurysms were created in 12 New Zealand White rabbits (body weight $3-4 \mathrm{~kg}$ ). Detailed procedures for aneurysm creation have been described in depth elsewhere. ${ }^{14}$ Aneurysms were permitted to mature for at least 3 weeks after creation. Rabbits were assigned to either coil or flow diverter treatment groups. Aneurysms were either embolized with platinum coils or treated with flow diverters (Pipeline Embolic Device, Covidien Inc, California, USA) placed in the parent artery, as previously described. ${ }^{15} 16$ Digital subtraction angiography (DSA) of the aortic arch was performed immediately before or after treatment. At 4 weeks following treatment, a follow-up DSA of the aortic arch was performed. The animals were then euthanized by using a lethal injection of pentobarbital. The aneurysms and the parent artery were harvested, longitudinally cut into two halves, and the samples were immediately snap frozen in liquid nitrogen and kept frozen at $-70^{\circ} \mathrm{C}$ until use. One half of the sample was used for gene expression and the other half was used for zymography analysis.

\section{Angiographic evaluation}

Images from DSA obtained immediately after device implantation and just before sacrifice were evaluated. Follow-up DSA images were assessed using a trichotomous scale (incomplete occlusion, near complete occlusion, or complete occlusion). 


\section{Quantitative real time PCR analysis}

The mRNA expression of selected genes that have been associated with vascular remodeling of aneurysms was assessed by quantitative real time PCR. These selected genes were categorized into cell adhesion (vascular cell adhesion molecule 1 (VCAM-1) and selectin E), structural molecules (type VIII collagen, type I collagen, and fibronectin), growth factors and cytokines (vascular endothelial growth factor 1 (VEGF-1), tumor necrosis factor $\alpha(\mathrm{TNF} \alpha)$, osteopontin, and monocyte chemoattractant protein 1 (MCP-1)), proteinases and their inhibitors (matrix metalloproteinase (MMP) 2 and 9, tissue inhibitor of MMP-1 and MMP-2 (TIMP-1 and TIMP-2), and cathepsin L), and cellular and oxidative stress markers (hypoxia inducible factor 1 (HIF-1), heme oxygenase 1 , endothelial nitric oxide synthase, von Willebrand factor, and smooth muscle actin). Briefly, total RNA was extracted from aneurysm tissue with RNeasy kit (Qiagen). First strand complementary DNAs were synthesized from $500 \mathrm{ng}$ of total RNA using a synthesis system (SuperScript III First-Strand Synthesis System; Invitrogen, Carlsbad, California, USA). Then real time PCR was performed with a cycler (iCycler; Bio-Rad, Hercules, California, USA) using SYBR Green PCR kit (Invitrogen). The specific primers were designed from corresponding sequences obtained from GenBank (the genetic sequence database of the National Institutes of Health, National Center for Biotechnology Information) using a Web tool (Primer 3; http://frodo.wi.mit. edu/primer $3 /) .{ }^{17}$

\section{Gelatin zymography for MMPs}

Soluble proteins were extracted from frozen samples. ${ }^{9}$ Protein samples were separated by $10 \%$ zymogram gel (Bio-Rad). Gels were washed with renaturation buffer (Bio-Rad) for $1 \mathrm{~h}$ and then incubated for $48 \mathrm{~h}$ at $37^{\circ} \mathrm{C}$ in development buffer (Bio-Rad). The gels were stained with $0.5 \%$ Coomassie blue R-250. The gelatinolytic bands, representing the activities of MMPs, were scanned and analyzed densitometrically using Licor software.

\section{Statistical analysis}

Genes with a fold change of 1.2 or more were considered upregulated whereas those with a fold change of 0.8 or less were considered downregulated. ${ }^{10}$

\section{RESULTS}

\section{Angiographic results}

Angiographic data are summarized in table 1 , and representative angiographic images are presented in figure 1 . There were no statistically significant differences in mean neck, width, and height between coil and flow diverter treated groups. Of the six aneurysms treated with flow diverter devices, at 4 weeks posttreatment, two aneurysms showed complete occlusion, two aneurysms showed near complete occlusion, and the remaining two aneurysms were patent, whereas all six aneurysms treated with platinum coils showed stable occlusion at 4 weeks post-

Table 1 Aneurysm geometry in the two treatment groups

\begin{tabular}{lll}
\hline & Flow diverter & Coil \\
\hline Neck width $(\mathrm{mm})$ & $3.7 \pm 0.9$ & $3.3 \pm 1.6$ \\
Width $(\mathrm{mm})$ & $4.5 \pm 1.4$ & $3.7 \pm 0.6$ \\
Height $(\mathrm{mm})$ & $9.4 \pm 2.5$ & $8.0 \pm 1.3$ \\
\hline
\end{tabular}

treatment. Representative angiographic images are presented in figure 1.

\section{Gene and protein expression results}

Cell adhesion and structural components

None of the adhesion molecules (VCAM and selectin E) showed differential expression between the flow diverter and coil treated aneurysms. Among the structural molecules studied, both collagen I (0.18-fold) and fibronectin (0.32-fold) showed remarkably decreased expression in the flow diverter treated aneurysms compared with the coiled aneurysms, whereas collagen VIII (0.82-fold) exhibited no significant change between the flow diverter and coil groups (table 2).

Growth factors, cytokines. and inflammatory molecules

The expression profiles of osteopontin (2.4-fold) and the proinflammatory genes TNF- $\alpha$ (1.3-fold) and MCP-1 (1.4-fold) were increased in aneurysms treated with flow diverters compared with aneurysms treated with platinum coils. VEGF expression (0.37-fold) was significantly less in the flow diverter treated samples.

Cellular and stress markers

Both the hypoxia marker, HIF-1 (0.42-fold), and the oxidative stress marker, heme oxygenase 1 (0.7-fold), were downregulated in flow diverted treated aneurysms compared with coiled
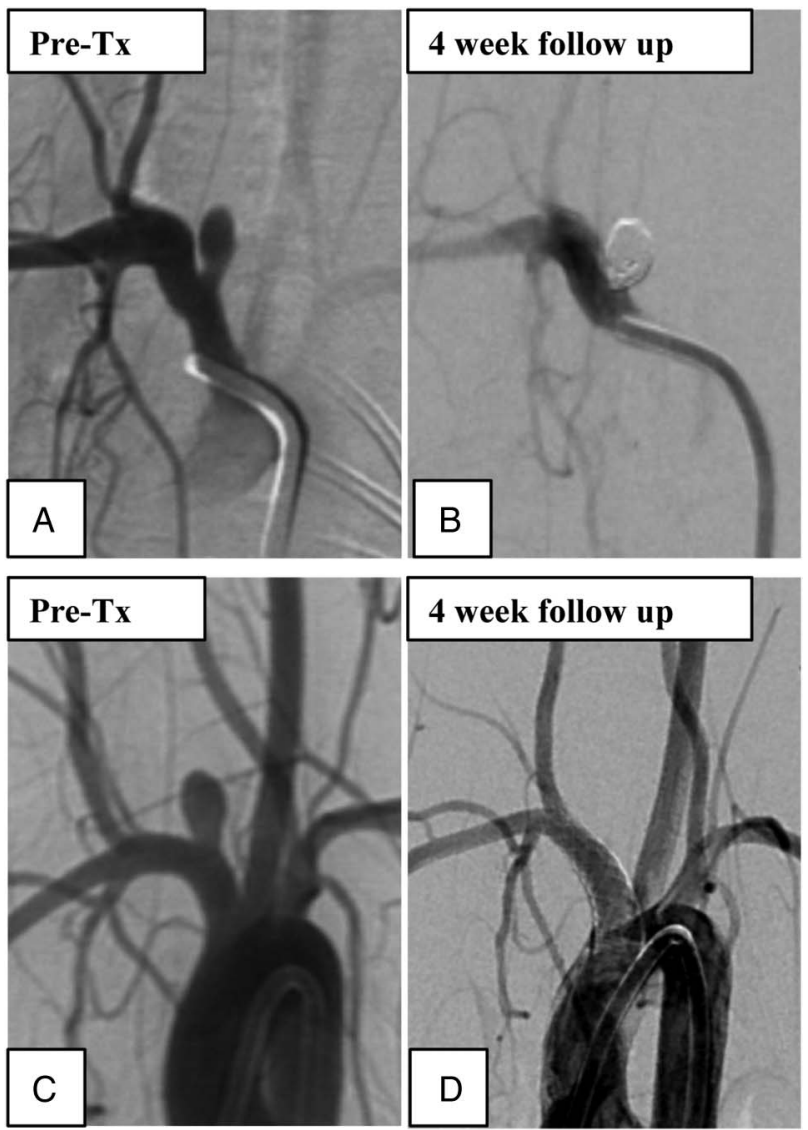

Figure 1 Representative angiograms of treated aneurysms. (A) Digital subtraction angiography (DSA) image showing the aneurysm cavity prior to coil treatment (Tx). (B) Follow-up DSA image showing obliteration of the aneurysm. (C) DSA image showing the aneurysm cavity prior to flow diverter placement. (D) Follow-up DSA image showing complete occlusion of the aneurysm. 
Table 2 Relative expression of genes in flow diverter treated aneurysms compared with coiled aneurysms

\begin{tabular}{|c|c|c|}
\hline Gene name & Median (range) & $\begin{array}{l}\text { Expression } \\
\text { changes }\end{array}$ \\
\hline \multicolumn{3}{|l|}{ Adhesion molecules } \\
\hline VCAM & $0.94(0.11-661.7)$ & No change \\
\hline Selectin E & $0.91(0.19-1.54)$ & No change \\
\hline \multicolumn{3}{|l|}{ Structural molecules } \\
\hline Collagen VIII & $0.82(0.07-180.39)$ & No change \\
\hline Collagen I & $0.18(0.06-1.38)$ & $\downarrow$ \\
\hline Fibronectin & $0.32(0.08-0.36)$ & $\downarrow$ \\
\hline \multicolumn{3}{|l|}{ Vascular remodeling } \\
\hline MMP-2 & $0.43(0.09-2.42)$ & $\downarrow$ \\
\hline MMP-9 & $0.36(0.02-94.03)$ & $\downarrow$ \\
\hline Cathepsin L & $0.25(0.07-1.1)$ & $\downarrow$ \\
\hline TIMP-1 & $2.4(0.11-1.13)$ & $\uparrow$ \\
\hline TIMP-2 & $0.18(0.02-2.51)$ & $\downarrow$ \\
\hline \multicolumn{3}{|c|}{ Growth factors/cytokines/inflammatory molecules } \\
\hline Osteopontin & $2.4(0.11-133.9)$ & $\uparrow$ \\
\hline $\begin{array}{l}\text { Monocyte chemoattractant } \\
\text { protein }\end{array}$ & $1.29(0.37-5.35)$ & $\uparrow$ \\
\hline TNF $\alpha$ & $1.37(0.63-27.19)$ & $\uparrow$ \\
\hline VEGF & $0.37(0.28-1.13)$ & $\downarrow$ \\
\hline \multicolumn{3}{|l|}{ Oxidative stress } \\
\hline HIF & $0.42(0.06-251.6)$ & $\downarrow$ \\
\hline HO-1 & $0.69(0.15-1.84)$ & $\downarrow$ \\
\hline \multicolumn{3}{|l|}{ Cellular markers } \\
\hline vWF & $0.35(0.12-0.14)$ & $\downarrow$ \\
\hline eNOS & $0.08(0.02-0.14)$ & $\downarrow$ \\
\hline SMA & $0.69(0.34-0.72)$ & $\downarrow$ \\
\hline
\end{tabular}

aneurysms. Significantly decreased expression of both endothelial cell makers, von Willebrand factor ( 0.35 -fold) and endothelial nitric oxide synthase (0.08), and the smooth muscle cell marker, smooth muscle actin (0.69-fold), was observed in the aneurysms of flow diverted treated animals.

\section{Proteases and their inhibitors}

Compared with flow diverter treated aneurysms, expression of MMP-2, MMP-9, and cathepsin L was upregulated over twofold in coil embolized aneurysms. TIMP-1 expression (2.4-fold) was elevated and TIMP-2 expression (0.2-fold) was diminished in the flow diverted treated group compared with the coiled group.

\section{MMP activities}

MMP zymography was performed to assess the activities of both inactive precursor and active functional forms of MMPs. Pro-MMP-2 expression was not different between the coil and flow diverted treated samples whereas active MMP-2 expression was $52 \%$ higher in coiled aneurysms. Pro-MMP-9 expression was diminished (66\%) while active MMP-9 expression was increased twofold in flow diverter treated aneurysms compared with coiled control aneurysm tissues (figure 2A, B).

\section{DISCUSSION}

In this study, we compared the gene expression pattern between aneurysms treated with diverters and aneurysms embolized with coils to probe the mechanism of aneurysm healing following two different interventional approaches. The major finding of this study was that active MMP-9 was involved in the remodeling of flow diverter treated aneurysms, as evidenced by an increase in the enzymatic activity of active MMP-9, despite a decrease in the level of mRNA. Our findings also demonstrated over expression of proinflammatory markers and under expression of structural genes and cellular markers in flow diverter treated aneurysms. There were no observed trends in the expression of VCAM-1 or selection E to suggest a role for adhesion molecules to account for flow diverter related remodeling.

The role of MMPs in the pathophysiology of cerebral aneurysms is well characterized, and they have also been reported in the recanalization and recurrence of canine aneurysms embolized with gelatin sponges. ${ }^{8}$ Similarly, high expression of MMP-9 has been associated with the healing of aneurysms following stent placement in the parent artery of a swine aneurysm model. ${ }^{7}$ Diminished expression of pro-MMP-2 and pro-MMP-9, seen in the zymogram, was correlated with decreased gene expression of the respective genes in the flow diverter treated group. Interestingly, profound elevation of active MMP-9 in the flow diverter treated aneurysms indicates a major role for MMP-9 in the remodeling of the aneurysm wall, which may be a possible explanation for the spontaneous rupture of aneurysms treated with flow diverters in patients. ${ }^{12}{ }^{13}$ Moreover, the activity of MMP-9 is important in cell migration of neointimal cells, and occlusion of aneurysms following flow diverters is thought to be derived from neointimal cell growth originating from the vessel wall. ${ }^{3}$ The observed increase in the activity of active MMP-9 in the flow diverter treated group could also be related to the ongoing vascular remodeling in the aneurysm cavity. The activity is more important in predicting the function of an enzyme that the level of mRNA. ${ }^{18}{ }^{19}$ Our previous study comparing the gene expression pattern between aneurysms with a high aspect (height to neck width) revealed high expression of hypoxia induced TIMP in high aspect ratio aneurysms. ${ }^{20}$ Similarly, in the present study, an increase in expression of TIMP-1 was observed in the flow diverter treated aneurysms. This could be related to active extracellular matrix turnover and vascular remodeling, which may lead to aneurysm rupture. ${ }^{21}$

TNF $\alpha$ is a proinflammatory cytokine with strong necrotic activity and is secreted by a variety of cells, including macrophages, endothelial cells, and smooth muscle cells. The chemokine MCP-1 recruits monocytes and neutrophils to the site of injury and promotes chemotaxis and inflammation. Deposition of inflammatory cells was noted in the struts of the flow diverters at the neck of the aneurysms. High levels of detected MCP- 1 and TNF $\alpha$ could be related to the inflammatory events in the aneurysm following flow diverter placement. ${ }^{3}$ Chow et $a l^{12}$ showed inflammatory infiltrates, mural thinning, and necrosis within the aneurysm of a delayed spontaneous rupture of a posterior inferior cerebellar artery aneurysm following treatment with flow diversion. An increase in the level of TNF $\alpha$ detected in this study may be associated with the inflammation and necrotic cell death of spontaneous rupture of flow diverter treated aneurysms. TNF has also been shown to increase MMP-9 activity. ${ }^{22}{ }^{23}$ Moreover, high levels of both TNF $\alpha$ and MMPs were reported in ruptured human cerebral aneurysms, ${ }^{24}$ and inhibition of TNF $\alpha$ has been shown to decrease the incidence of aneurysm rupture in a mouse model. ${ }^{25}$ The observed elevated levels of HIF and VEGF in coiled aneurysms could be related to the neovascularization of thrombus in the aneur$\mathrm{ysm}^{2627}$ as the geometry of untreated rabbit aneurysms remains stable for up to 5 years. ${ }^{28}$ 
Figure 2 (A) Matrix metalloproteinase (MMP)-2 and MMP-9 enzymatic activities of aneurysm samples at 4 weeks following flow diverter and coil embolization on gelatin zymography.

(B) Densitometry analysis of activities of pro- and active MMP-2 and MMP-9.
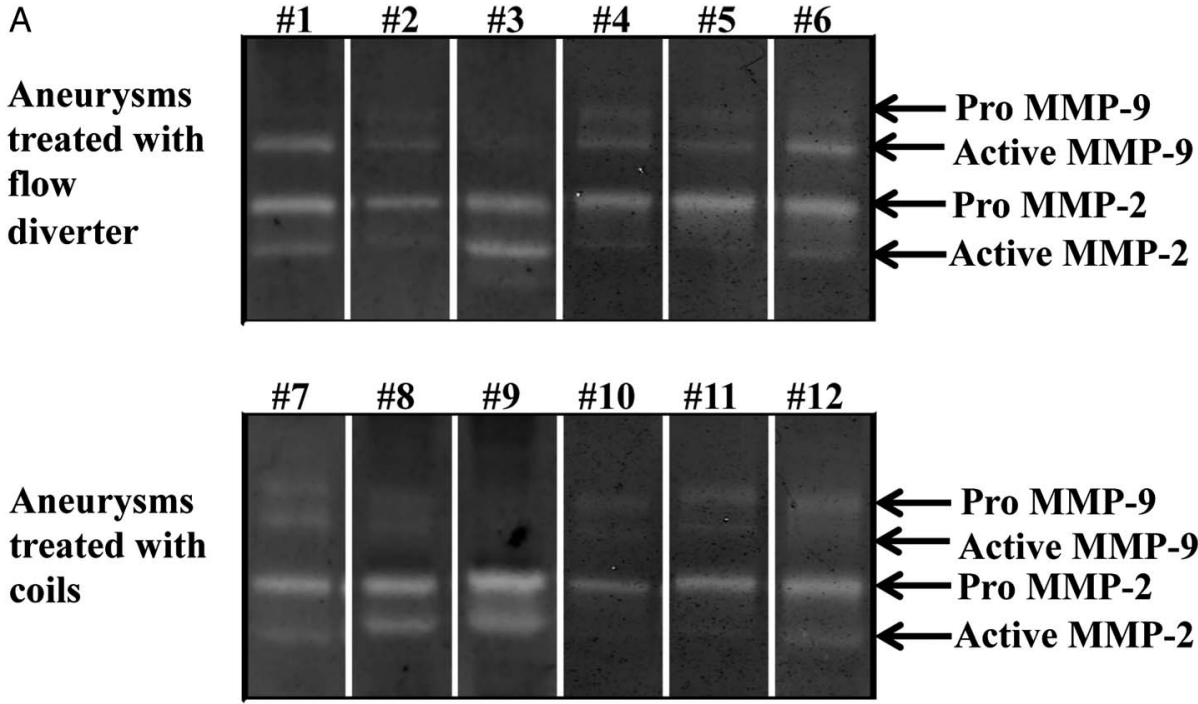

B

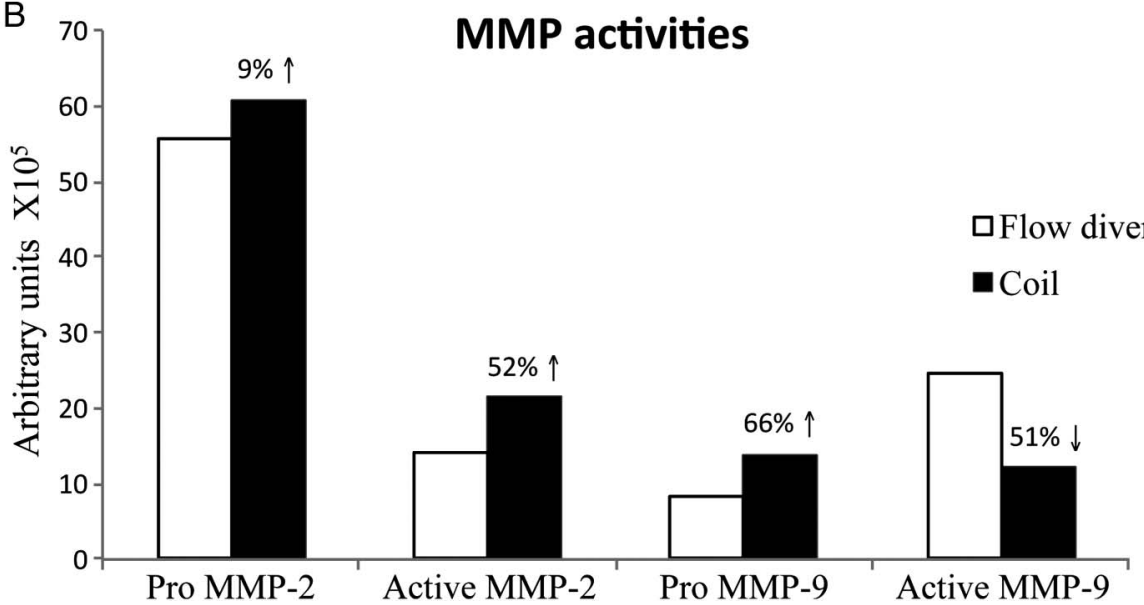

Our study had several limitations. The flow diverter and coil treated groups were not compared with untreated aneurysms or normal control arteries. We assessed gene expression of completely occluded coiled aneurysms against incompletely occluded flow diverter treated aneurysms. In addition, we compared differences between complete occlusion, near complete occlusion, and patent groups. Even though the rabbit aneurysm model employed in this study is similar to human aneurysms histologically, ${ }^{29}$ morphologically, ${ }^{30}$ biologically, ${ }^{31}$ and hemodynamically, ${ }^{32}$ further studies on human samples will be necessary to validate our findings. We only studied one time point following embolization, and gene expression may change over time.

\section{CONCLUSION}

In the rabbit saccular aneurysm model, active MMP-9 activity and genes involved in the production of proteins that function under inflammation were upregulated whereas genes coding for cellular markers and structural proteins were downregulated. These findings may be helpful towards further understanding of the molecular pathways involved in the healing of aneurysms treated with either flow diverter devices or platinum coils.

Acknowledgements We thank Covidien Inc for generously providing the flow diverters for this study.

Contributors CP: gene expression experiments, statistical analysis, and manuscript drafting. DD and Y-HD: animal experiments, including aneurysm creation, embolization, and follow-up, and tissue harvest. JC, DK, and RK: study design, data analysis, and manuscript editing. All of the authors have read and approved submission of the manuscript.

Funding This work was supported by National Institutes of Health grant NS 076491.

Competing interests None.

Provenance and peer review Not commissioned; externally peer reviewed.

\section{REFERENCES}

1 Ribourtout E, Raymond J. Gene therapy and endovascular treatment of intracranial aneurysms. Stroke 2004;35:786-93.

2 Mitome-Mishima Y, Yamamoto M, Yatomi K, et al. Endothelial cell proliferation in swine experimental aneurysm after coil embolization. PLOS ONE 2014;9:e89047.

3 Kadirvel R, Ding YH, Dai D, et al. Cellular mechanisms of aneurysm occlusion after treatment with a flow diverter. Radiology 2014;270:394-9.

4 Li ZF, Fang XG, Yang PF, et al. Endothelial progenitor cells contribute to neointima formation in rabbit elastase-induced aneurysm after flow diverter treatment. CNS Neurosci Ther 2013;19:352-7.

5 Guglielmi G, Vinuela F, Dion J, et al. Electrothrombosis of saccular aneurysms via endovascular approach. Part 2: Preliminary clinical experience. J Neurosurg 1991;75:8-14.

6 D'Urso Pl, Lanzino $\mathrm{G}, \mathrm{Cloft} \mathrm{HJ}$, et al. Flow diversion for intracranial aneurysms: a review. Stroke 2011;42:2363-8.

7 Bouzeghrane F, Darsaut T, Salazkin I, et al. Matrix metalloproteinase-9 may play a role in recanalization and recurrence after therapeutic embolization of aneurysms or arteries. J Vasc Interv Radiol 2007;18:1271-9.

8 Darsaut T, Salazkin I, Ogoudikpe C, et al. Effects of stenting the parent artery on aneurysm filling and gene expression of various potential factors involved in healing of experimental aneurysms. Interv Neuroradiol 2006;12:289-302. 
9 Kadirvel R, Ding YH, Dai D, et al. Molecular indices of apoptosis activation in elastase-induced aneurysms after embolization with platinum coils. Stroke 2007;38:2787-94.

10 Kadirvel R, Ding YH, Dai D, et al. Differential gene expression in well-healed and poorly healed experimental aneurysms after coil treatment. Radiology 2010;257:418-26.

11 Kadirvel R, Ding YH, Dai D, et al. Proteomic analysis of aneurysm healing mechanism after coil embolization: comparison of dense packing with loose packing. AJNR Am J Neuroradiol 2012;33:1177-81.

12 Chow M, McDougall C, O'Kelly C, et al. Delayed spontaneous rupture of a posterior inferior cerebellar artery aneurysm following treatment with flow diversion: a clinicopathologic study. AJNR Am J Neuroradiol 2012;33:E46-51.

13 Kulcsar Z, Houdart E, Bonafe A, et al. Intra-aneurysmal thrombosis as a possible cause of delayed aneurysm rupture after flow-diversion treatment. AJNR Am J Neuroradiol 2011;32:20-5.

14 Altes TA, Cloft HJ, Short JG, et al. 1999 ARRS Executive Council Award. Creation of saccular aneurysms in the rabbit: a model suitable for testing endovascular devices. American Roentgen Ray Society. AJR Am J Roentgenol 2000;174:349-54.

15 Kallmes DF, Ding YH, Dai D, et al. A new endoluminal, flow-disrupting device for treatment of saccular aneurysms. Stroke 2007;38:2346-52.

16 Kallmes DF, Helm GA, Hudson SB, et al. Histologic evaluation of platinum coil embolization in an aneurysm model in rabbits. Radiology 1999; 213:217-22.

17 Rozen S, Skaletsky H. Primer3 on the WWW for general users and for biologist programmers. Methods Mol Biol 2000;132:365-86.

18 Laurent JM, Vogel C, Kwon T, et al. Protein abundances are more conserved than mRNA abundances across diverse taxa. Proteomics 2010;10:4209-12.

19 Svensson C, Welsh N, Krawetz SA, et al. Exhibition of specific alterations in activities and mRNA levels of rat islet glycolytic and mitochondrial enzymes in three different in vitro model systems for attenuated insulin release. Diabetes 1991;40:771-6.

20 Kadirvel R, Ding YH, Dai D, et al. Differential expression of genes in elastase-induced saccular aneurysms with high and low aspect ratios. Neurosurgery 2010;66:578-84; discussion 84.
21 Jin D, Sheng J, Yang $X$, et al. Matrix metalloproteinases and tissue inhibitors of metalloproteinases expression in human cerebral ruptured and unruptured aneurysm. Surg Neurol 2007;68(Suppl 2):S11-16; discussion S16.

22 Han SJ, Hawkins SM, Begum K, et al. A new isoform of steroid receptor coactivator-1 is crucial for pathogenic progression of endometriosis. Nat Med 2012;18:1102-11.

23 Tsai CL, Chen WC, Hsieh HL, et al. TNF-alpha induces matrix metalloproteinase-9-dependent soluble intercellular adhesion molecule-1 release via TRAF2-mediated MAPKs and NF-kappaB activation in osteoblast-like MC3T3-E1 cells. J Biomed Sci 2014;21:12.

24 Jayaraman T, Paget A, Shin YS, et al. TNF-alpha-mediated inflammation in cerebral aneurysms: a potential link to growth and rupture. Vasc Health Risk Manag 2008:4:805-17.

25 Starke RM, Chalouhi N, Jabbour PM, et al. Critical role of TNF-alpha in cerebral aneurysm formation and progression to rupture. J Neuroinflammation 2014;11:77.

$26 \operatorname{Lim}$ CS, Kiriakidis S, Sandison A, et al. Hypoxia-inducible factor pathway and diseases of the vascular wall. J Vasc Surg 2013;58:219-30.

27 Abrahams JM, Forman MS, Grady MS, et al. Delivery of human vascular endothelial growth factor with platinum coils enhances wall thickening and coil impregnation in a rat aneurysm model. AJNR Am J Neuroradiol 2001;22:1410-17.

28 Ding Y, Dai D, Kadirvel R, et al. Five-year follow-up in elastase-induced aneurysms in rabbits. AJNR Am J Neuroradiol 2010;31:1236-9.

29 Dai D, Ding YH, Danielson MA, et al. Histopathologic and immunohistochemical comparison of human, rabbit, and swine aneurysms embolized with platinum coils. AJNR Am J Neuroradiol 2005;26:2560-8.

30 Short JG, Fujiwara NH, Marx WF, et al. Elastase-induced saccular aneurysms in rabbits: comparison of geometric features with those of human aneurysms. AJNR Am J Neuroradiol 2001;22:1833-7.

31 Mangrum WI, Farassati F, Kadirvel R, et al. mRNA expression in rabbit experimental aneurysms: a study using gene chip microarrays. AJNR Am J Neuroradiol 2007;28:864-9.

32 Zeng Z, Kallmes DF, Durka MJ, et al. Hemodynamics and anatomy of elastase-induced rabbit aneurysm models: similarity to human cerebral aneurysms? AJNR Am J Neuroradiol 2011;32:595-601. 